\title{
DEFINIÇÃO DO INTERVALO ÓTIMO DE INSPEÇÕES COM BASE NO CONCEITO DELAY TIME: UMA APLICAÇÃO EM UMA UNIDADE INDUSTRIAL DE PRODUÇÃO CONTÍNUA
}

\author{
Glauco Ricardo Simões Gomes \\ Universidade Federal da Paraíba (UFPB) \\ Observatório de Pesquisa e Prática em Administração (OPPA) \\ Campus Universitário, Centro de Ciências Sociais Aplicadas, Bloco A, Sala 217 \\ João Pessoa - PB, 58051-900 \\ glaucorsg@gmail.com
}

\begin{abstract}
Resumo
O presente trabalho apresenta uma aplicação de modelagem matemática orientada à gestão da manutenção que tem como base o conceito delay time. O cenário do estudo foi a área de produção de uma unidade industrial de fluxo contínuo que opera 24 horas por dia. A ideia principal foi a de utilizar os conceitos desta abordagem para determinar os tempos ótimos das ações preventivas do departamento de manutenção de modo a garantir uma maior disponibilidade dos equipamentos e instalações enquanto apresenta custos adequados de intervenção da área de manutenção. Após uma breve introdução sobre o tema (seção 1), o artigo apresenta na seção 2, tópicos que ilustram e ressaltam a importância da modelagem matemática aplicada à gestão da manutenção e do conceito delay time. A sessão 3 traz as características da empresa onde o estudo foi realizado, assim como os dados referentes ao processo produtivo e às ações de manutenção. A seção 4 apresenta os resultados obtidos após a aplicação do conceito delay time e por fim, estes resultados são discutidos bem como as limitações do artigo e as propostas de trabalhos futuros (seção 5).

Palavras-chaves: Planejamento da manutenção; delay time; modelagem matemática, custo, disponibilidade.

Abstract

This paper presents an application of mathematical modeling oriented to maintenance management based on the delay time concept. The study scenario was the manufacturing sector of an industrial unit which operates 24 hours a day in continuum flux of production. The main idea was to use the concepts of this approach to determine the optimal time of preventive action from the maintenance department to ensure greater availability of equipment and facilities while introducing appropriate maintenance costs. After a brief introduction on the subject (section 1), the article presents in section 2, topics that illustrate and underscore the importance of mathematical modeling applied to the maintenance management and delay time concept. Session 3 shows the characteristics of the company where the study was conducted, as well as data relating to the production process and maintenance actions. Section 4 presents the results obtained after applying the delay time concept and finally, these results are discussed as well as the limitations of the article and the proposals for future work (section 5).
\end{abstract}

Keywords: Maintenance planning; delay time; mathematical modeling, cost, availability. 


\section{INTRODUÇÃO}

Empresas de base industrial quer sejam de pequeno, médio ou grande porte, enfrentam o desafio de se manterem cada vez mais competitivas em um mercado globalizado e voltado a políticas de redução de custos. Fato é, que para realizar tais reduções, buscam-se diferenciais em seus processos internos e externos. Entretanto, procedimentos como a aquisição de materiais e compra de equipamentos com melhor rendimento estão facilmente acessíveis a todos os concorrentes e com isso, deixam de figurar como uma exclusividade.

Assim sendo, fatores internos às operações evidenciam-se como o verdadeiro diferencial competitivo das organizações que pretendem manter-se no mercado. Neste sentido, Tsang (2002) ressalta que a manutenção apresenta-se como uma função de suporte para aquelas empresas com forte investimento em equipamentos e instalações. Além disso, a manutenção tem papel fundamental na contribuição da melhoria da competitividade visto que garante uma melhor utilização dos equipamentos orientada aos objetivos da empresa.

Diversos autores como Alsyouf (2007), Eti et al. (2006) e Murthy (2002), enaltecem o papel do planejamento da manutenção como elemento de diferencial competitivo, por ter por objetivo a geração de ações que garantem a disponibilidade dos equipamentos e instalações durante o seu processo natural de degradação, não só impactando no desempenho do negócio, mas também na qualidade de produtos e serviços.

$\mathrm{Na}$ tentativa de harmonizar as demandas operacionais e de manutenção, o planejamento tem que lidar com questões cruciais e muitas vezes conflitantes; envolvendo, por exemplo, decisões sobre quando realizar uma inspeção, quando desativar um equipamento para uma manutenção ou qual a idade ótima para a substituição de componente. Conforme cresce a importância da área de manutenção, cresce também a necessidade de implementações de políticas de manutenção de forma a suportar tais questões (WANG, 2002).

A modelagem matemática completa este cenário figurando como ferramenta para um melhor processo de planejamento das ações de manutenção levando em consideração tanto seus custos quanto os benefícios associados; objetivando assim, um equilíbrio entre os mesmos (DEKKER, 1996).

Apesar da sua grande aplicabilidade, Scarf (1997) elucida que os modelos matemáticos orientados à gestão da manutenção deparam-se com um grande desafio: o sucesso da área de modelagem só pode ser medido em termos do sucesso na resolução de problemas reais de manutenção. Assim, fica clara a necessidade de uma aderência destes modelos e de seus respectivos resultados à realidade das empresas. Desta forma, o objetivo seria o de modelar o problema de maneira a obter-se políticas ótimas de manutenção para uma posterior aplicação e que venha a trazer benefícios de curto prazo para as organizações.

Dentre as modelagens mais tradicionais da área de manutenção, destaca-se o conceito delay time que aborda a interrupção do funcionamento do equipamento ou sistema como um processo de dois estágios, onde inicialmente existem indícios de um defeito latente e um intervalo de tempo hábil onde pode-se tomar uma ação previamente à efetiva falha do sistema.

O presente trabalho apresenta uma aplicação de modelagem matemática orientada à gestão da manutenção que tem como base o conceito delay time. A ideia principal é a de utilizar os conceitos desta abordagem para determinar os tempos ótimos das ações preventivas de um departamento de manutenção de modo a garantir uma maior disponibilidade dos equipamentos e instalações enquanto apresenta custos adequados de intervenção da área de manutenção.

O artigo está dividido da seguinte forma: após a introdução referente ao tópico 1, apresenta-se um breve discussão acerca da modelagem matemática em manutenção e em particular a abordagem delay time (tópicos 2 e 2.1). A terceira parte do trabalho refere-se à contextualização do problema e apresentação dos dados utilizados para ilustrar a aplicação 
dos modelos. Posteriormente, apresentam-se os resultados obtidos (e suas respectivas considerações) e as referências utilizadas.

\section{MODELAGEM MATEMÁTICA APLICADA À MANUTENÇÃO}

Segundo Levitt (1997), a função manutenção busca otimizar o uso dos ativos de uma organização ao longo do seu ciclo de vida; o que significa não só preservá-los, como também preservar sua capacidade de produção em níveis aceitáveis de segurança e rentabilidade.

Ainda segundo o autor, a gestão da manutenção apresenta-se hoje como uma função importante e complexa, visto o grande número de custos operacionais e de suporte que incorrem sobre equipamentos e sistemas.

Para Figueiredo et al. (2008), à gestão da manutenção (e seu consequente planejamento) estão associados três tipos de ações de manutenção - corretiva, preventiva e preditiva. A manutenção corretiva tem natureza reativa, pois é realizada após a ocorrência da falha do equipamento ou sistema; já as manutenções preventivas e preditivas têm caráter proativo, visto que são realizadas previamente à falha e atuam de maneira a diminuir a probabilidade de ocorrência deste evento.

Levitt (2003) ressalta que apesar de não ser a prática dominante, existem duas situações onde a manutenção preventiva é importante: quando esta reduz o risco ou a probabilidade de mortes, lesões ou danos ambientais ou quando o custo da atividade de manutenção é inferior ao custo da consequência da sua não realização.

Neste sentido, destacam-se duas importantes ferramentas para o suporte da gestão da manutenção. O conceito delay time que determina intervalos ótimos para inspeção de sistemas sujeitos a defeitos que futuramente levarão a falhas; e a noção de manutenção imperfeita, onde admite-se que as ações de manutenção carregam em si uma parcela de "não qualidade" e que podem levar o sistema a interrupções antes do previsto.

\subsection{O CONCEITO DELAY TIME}

O conceito de delay time foi originalmente proposto por A. H. Christer em 1982 no artigo "Modelling inspection policies for building maintenance" para a resolução de problemas de manutenção em edificações. Posteriormente o conceito foi aplicado em ambientes de manutenção industrial e desde então vem sendo amplamente utilizado (BAKER e CHRISTER, 1994).

Segundo os autores supracitados, o conceito delay time tem como motivação a representação dos outputs do processo de manutenção enquanto variáveis de decisão para a gerência. Mais especificamente, consiste em determinar o melhor momento para intervir em um equipamento ou sistema dado o aparecimento de um defeito, antes da sua falha total.

Caracterizando-se como uma modelagem bastante pragmática, o conceito foi prontamente adotado por engenheiros de maneira a modelar problemas de decisão acerca de inspeções periódicas em sistemas sujeitos a processos de degradação (CHRISTER e WALLER, 1984; CHRISTER et al., 1995; CHRISTER, 1999; WANG, 2003).

$\mathrm{O}$ conceito delay time aborda o evento falha como um processo de dois estágios; desta feita, considera-se um intervalo de tempo h que reflete o período entre o surgimento de um defeito e a efetiva ocorrência de uma falha causada por este. O objetivo então é modelar o problema de maneira a obter melhores valores para $h$ para que com isso, sejam estabelecidas estratégias ótimas de manutenção (JONES et al, 2009; WANG e JIA, 2007).

A figura 1 ilustra o conceito de delay time.

Figura 1 - Ilustração do conceito delay time 


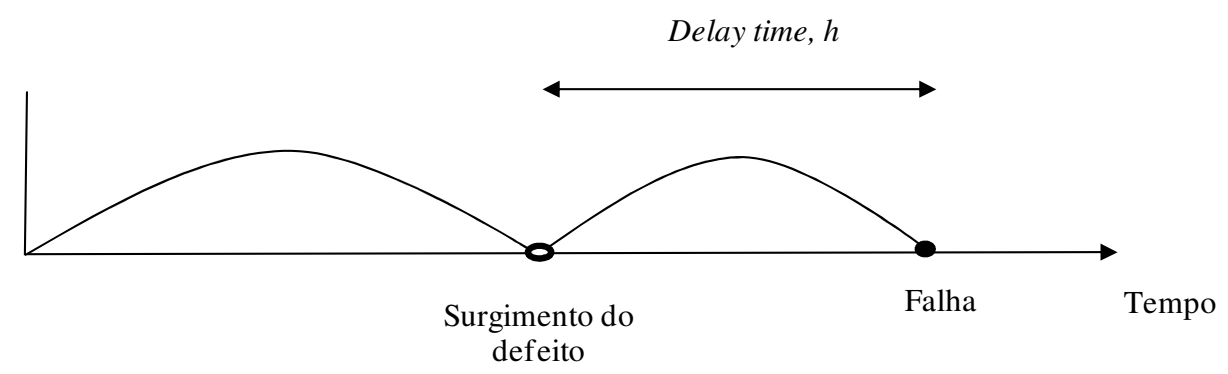

Christer et al. (1998) definem o delay time como uma extensão do tradicional conceito de tempo até a falha já difundido nos estudos de confiabilidade. Entretanto, a diferencia-se o fato de que caso o objeto de estudo (equipamento ou sistema) tenha sido inspecionado previamente, pode-se evidenciar a possibilidade de ocorrência de uma falha futura; e com isso, uma tratativa de prevenção pode ser adotada.

Mowbray (1997) ressalta que a existência de um período entre o surgimento de um defeito observável e a parada não planejada do sistema (breakdown) não se mostra como algo inédito para os engenheiros; haja vista as já difundidas implementações de políticas de manutenção preventiva, sistemas de inspeção e o conceito de Manutenção Centrada na Confiabilidade (MCC).

Para Wang e Jia (2007), o diferencial do delay time estaria então na tentativa de capturar quantitativamente, a relação entre a evidência de um defeito e a ocorrência da falha e, explorá-la para a construção das rotinas de inspeção.

Para Wang (1997), apesar de $h$ normalmente não ser mensurável, foi provada a possibilidade da sua probabilidade ser estimada tanto por meios objetivos como subjetivos.

Uma tratativa tradicional na construção dos modelos que fazem uso do delay time é a adoção de algumas suposições que ilustram as condições de contorno do sistema estudado. Baker e Christer (1994) listam as suposições gerais que caracterizam o conceito delay time:

a) Uma falha é detectada tão logo ela ocorra e sem a necessidade de inspeção;

b) Um sistema que apresente falha deve ser reparado antes de voltar à operação;

c) Antes da ocorrência da falha, um componente passa por um ou mais estados defeituosos;

d) A detecção de um estado defeituoso de um componente só pode ser realizada através de uma inspeção.

Conforme citado previamente, o conceito delay time apresenta-se como uma válida ferramenta para o processo de decisão da gestão da manutenção e cujo principal "produto" é a definição de políticas de manutenção estruturadas. Alguns dos parâmetros comumente discutidos nessas aplicações são discutidos por Ferreira et al. (2009) e apresentados nas equações 1, 2 e 3 abaixo:

- A probabilidade de ocorrência de uma falha gerar parada total do sistema (breakdown)

$$
b(t)=\int_{0}^{t}\left(\frac{t-h}{t}\right) f(h) d h
$$

- O custo esperado política de inspeção periódica

$$
C(t)=\frac{\lambda t\left\{C_{b} b(t)+C_{r}[1-b(t)]\right\}+C_{i}}{(t+d)}
$$

- O tempo inativo (downtime) gerado por esta política

$$
D(t)=\frac{\lambda t d_{b} b(t)+d}{(t+d)}
$$


Onde $t$ é o intervalo de tempo entre as inspeções; $\lambda$ é a taxa de chegada de defeitos por unidade de tempo; $f(h)$ é a função densidade de probabilidade do delay time; $C_{b}, C_{r}$ e $C_{i}$ são, respectivamente, os custos de breakdown, reparo e inspeção; e $d_{b}$ e $d_{i}$ são respectivamente os tempos médios de breakdown e de inspeção.

Jones, Jenkinson e Wang (2009) apresentam uma metodologia para aplicação do conceito delay time em ambientes industriais de manufatura. Neste trabalho, os autores apresentam uma abordagem um pouco mais sutil que prevê uma diferenciação entre as taxas de chegadas de defeitos $\left(k_{f}\right)$ e falhas $(\lambda)$. A diferenciação destes dois parâmetros é refletida em novas equações para os modelos de downtime e custos (equações 4 e 5). A equação para a probabilidade de parada total do sistema (breakdown) se mantém similar àquela apresentada anteriormente (equação 1). As equações podem ser visualizadas abaixo:

$$
\begin{aligned}
& D(t)=\left\{\frac{d_{i}+k_{f} t b(t) d_{b}}{t+d_{i}}\right\} \\
& C(t)=\frac{\left[k_{f} t\left\{C_{b} b(t)+C_{r}[1-b(t)]\right\}+C_{i}\right]}{\left(t+d_{i}\right)}
\end{aligned}
$$

Onde:

$f(h)$ - função distribuição de probabilidade acumulada que representa o comportamento do delay time no sistema.

$\mathrm{d}_{\mathrm{i}}$ - duração de uma atividade de inspeção;

$\mathrm{d}_{\mathrm{b}}$ - duração de uma falha ocasionada por um breakdown;

$\mathrm{C}_{\mathrm{b}}$ - custo de uma falha ocasionada por breakdown;

$\mathrm{C}_{\mathrm{r}}$ - custo da realização de um reparo detectado em uma inspeção;

$\mathrm{C}_{\mathrm{i}}$ - custo de realização de uma inspeção;

$k_{f}$ - taxa de chegada de defeitos ao sistema;

$\lambda(t)$ - taxa de chegada de falhas ao sistema.

\section{CONTEXTUALIZAÇÃO DO PROBLEMA}

De forma a ilustrar a aplicabilidade dos conceitos do delay time e da manutenção imperfeita, apresenta-se aqui um problema de definição de intervalos ótimos de inspeções de modo garantir uma maior eficácia do sistema estudado. Eficácia esta, refletida através dos parâmetros de downtime, custo da política preventiva e disponibilidade total do sistema.

O problema tem como base os dados referentes à taxa de falhas, duração e custos de ações de manutenção e a qualidade das inspeções realizadas em um processo de produção contínuo que foi observado por cerca de 4 meses.

O processo opera em um regime de 24 horas por dia, 7 dias por semana. A cada dois meses, uma manutenção preventiva com duração de 3 dias é executada onde os principais problemas apontados pela área de produção são tratados. Manutenções corretivas (emergenciais) são realizadas sempre que o processo é interrompido em função de uma falha.

A motivação para a escolha desse processo (além do acesso às informações) foi a alta taxa de manutenções corretivas encontrada combinada com sua natureza contínua que ressalta a necessidade de inspeções de qualidade (visto que o processo não pode ser interrompido com frequência para eventuais intervenções).

A partir das informações fornecidas pela empresa e das observações in loco pode-se verificar que o sistema apresenta uma taxa de chegada de 0,4625 de defeitos por hora, que por sua vez, desdobram-se em 0,0960 falhas por hora. Os dados de falha coletados junto à supervisão de manutenção foram posteriormente inseridos no módulo Input Analyzer do 
simulador ARENA $^{\circledR}$ versão 12.0 onde puderam ser realizados testes de aderência para a distribuição de probabilidade de falhas e do delay time.

Ao final deste processo, verificou-se que a distribuição de probabilidade para as falhas pode ser representada por uma distribuição exponencial negativa e o delay time por uma distribuição de Weibull com parâmetro de forma $\beta$ igual a 7,114. As informações referente aos custos foram extraídas junto ao sistema ERP utilizado para a gestão dos diversos processos da empresa e então validados junto à equipe de controladoria da unidade. Os dados do processo são apresentados no quadro 1:

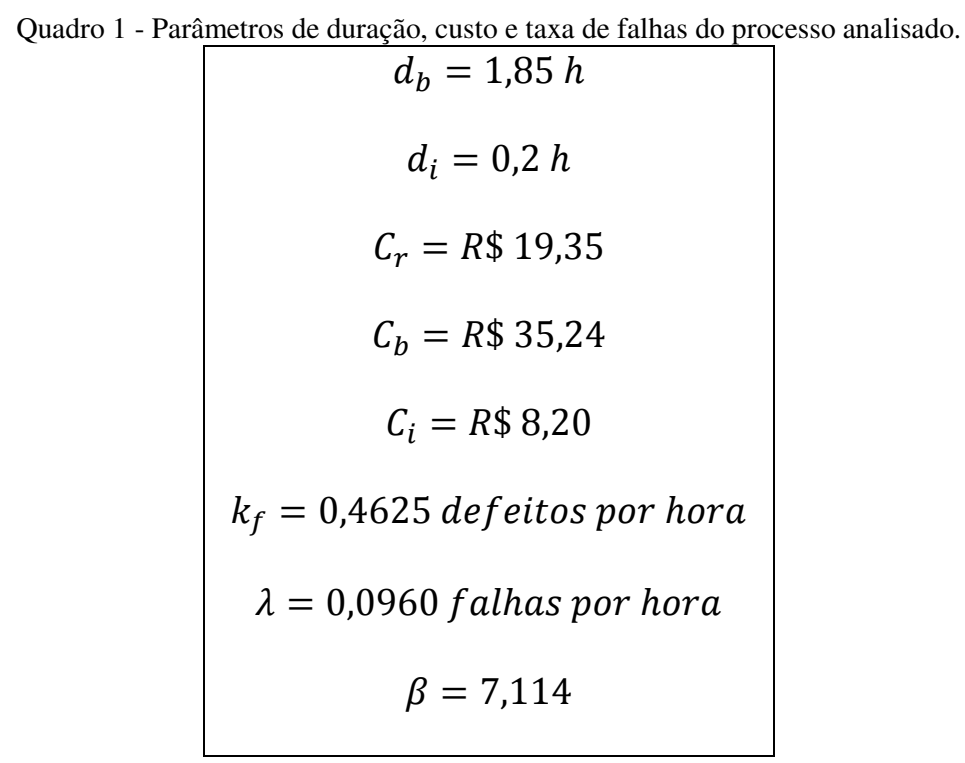

\section{RESULTADOS}

Após a o levantamento e tratamento dos dados do processo, o próximo passo foi a implantação do modelo contendo as formulações desenvolvidas por Jones, Jenkinson e Wang (2009) de forma a obter o período ótimo de inspeções dentro de um intervalo de 720 horas (um mês de trabalho). O modelo foi implementado com o auxílio do software Mathcad ${ }^{\circledR} 14.0$ e os respectivos gráficos ilustrando o comportamento de cada um dos parâmetros de interesse são apresentados a seguir.

De forma a tornar os resultados mais apresentáveis, calculou-se adicionalmente a disponibilidade do sistema ao longo do tempo conforme apresentado por Nascimento Neto et al. (2012). Percebe-se nesta formulação, a necessidade do parâmetro $d_{r}$ que representa a duração média de um reparo a ser realizado frente à parada do sistema. Para o nosso exemplo, temos $d_{r}=0,82$.

$$
\mathrm{A}(\mathrm{t})=\frac{720-\left(\left(\frac{720 * \mathrm{~d}_{\mathrm{i}}}{\mathrm{t}}\right)+\left(\mathrm{b}(\mathrm{t}) * \mathrm{~d}_{\mathrm{b}}\right)+\left(\frac{720 * \mathrm{~d}_{\mathrm{r}}}{\mathrm{t}} * \lambda\right)\right)}{720}
$$

A partir das análises realizadas, observou-se que o momento mais propício para a realização de intervenções programadas de manutenção deveria se dar no começo das operações e posteriormente com certa frequência. Particularmente, pode-se perceber a partir das figuras 2 e 3 que à medida em que o tempo de operação aumenta, temos uma maior probabilidade de ocorrência de parada total do sistema (breakdown) e um aumento do tempo de parada do sistema (downtime).

Figura 2 - Breakdown esperado ao longo do tempo 


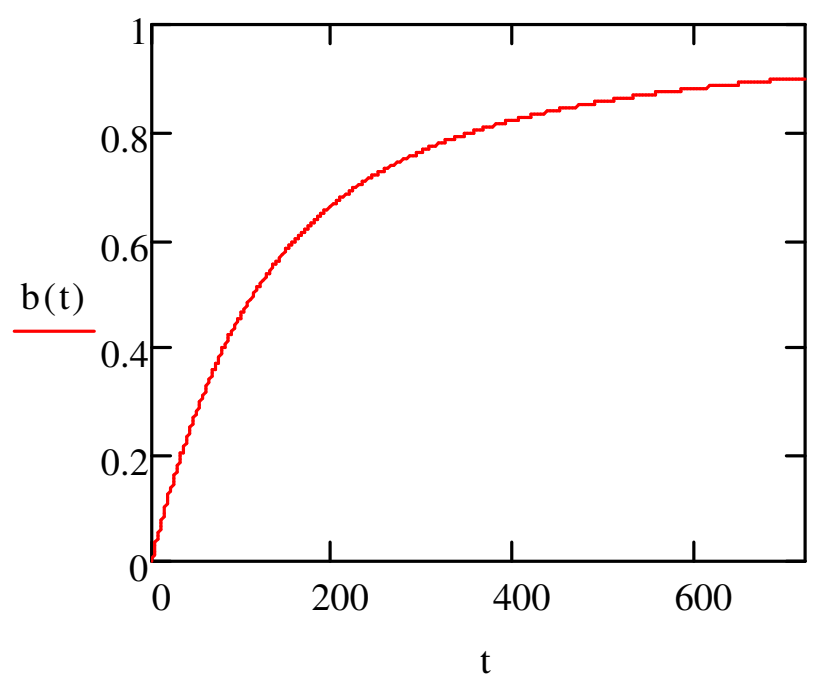

Figura 3 - Downtime esperado por unidade ao longo do tempo

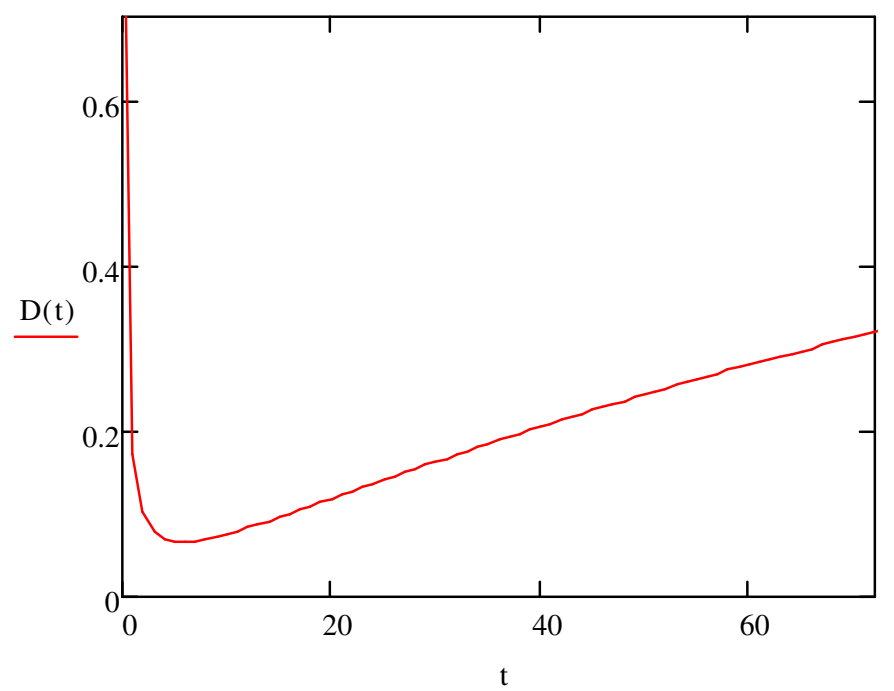

Analogamente, analisam-se os parâmetros de custo - $\operatorname{Cost}(t)$ - e disponibilidade do sistema - $A(t)$. Em consonância com as análises anteriores, percebe-se que os parâmetros de custos (figura 4) e disponibilidade (figura 5) acompanham o comportamento do sistema até aqui. Contudo, para o caso da disponibilidade, observa-se que após apresentar um pico no início da operação, tem-se uma queda nos valores de disponibilidade em função do próprio desgaste dos componentes frente à operação ininterrupta adotada pela empresa e uma posterior estabilidade abaixo do valor ótimo. 
Figura 4 - Custo esperado por unidade de tempo

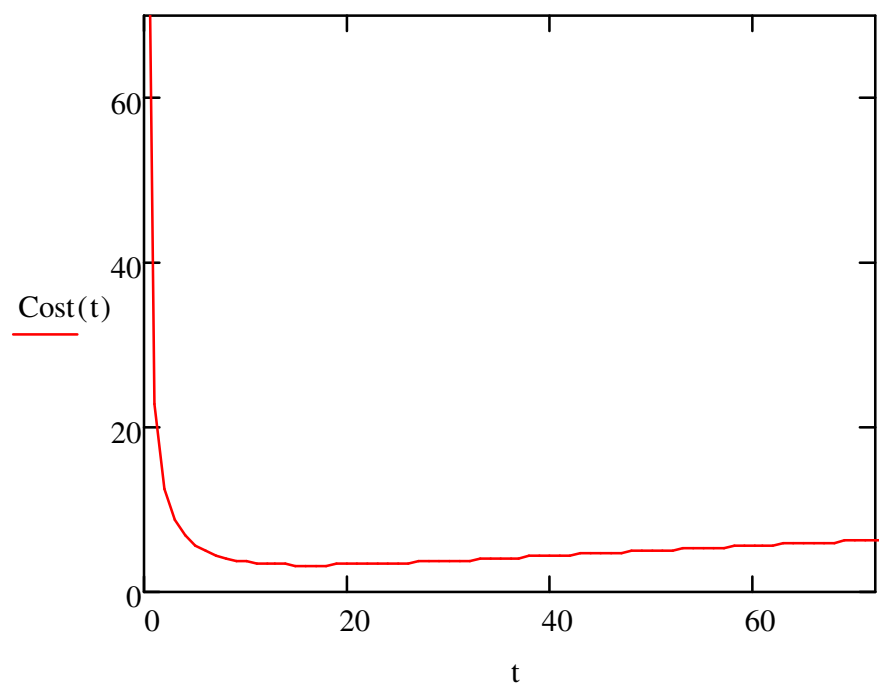

Figura 5 - Disponibilidade esperada ao longo do tempo

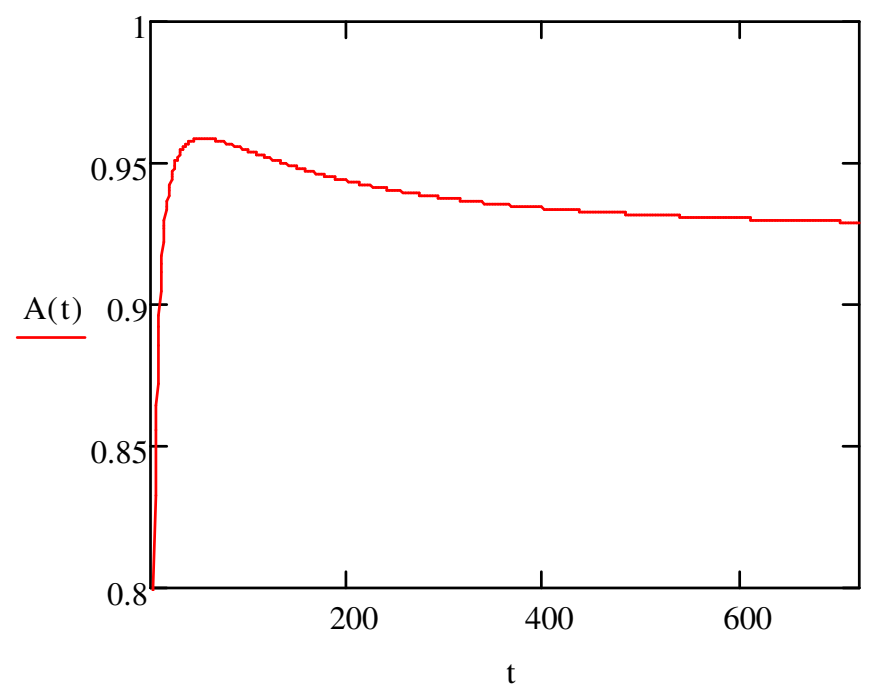

Após a análise gráfica dos parâmetros, resta então definir qual o intervalo ótimo $t^{*}$ para a realização destas inspeções. Para tanto, analisou-se os pontos de inflexão das funções que representam os parâmetros estudados (máximos ou mínimos dependendo do parâmetro em questão). Através da minimização dos vetores $\mathrm{D}(\mathrm{t})$ e $\operatorname{Cost}(\mathrm{t})$ e da maximização do vetor $\mathrm{A}(\mathrm{t})$ pode-se chegar aos valores ótimos para os intervalos de inspeção segundo cada um desses critérios. A tabela 1 apresenta tais resultados. 
Tabela 1 - Resultados após a otimização dos modelos

\begin{tabular}{cccc}
\hline \multicolumn{4}{c}{ Tabela 1 - Resultados após a otimização dos modelos } \\
\hline Parâmetro & Função & Tempo ótimo (t*) & $\begin{array}{c}\text { Valor ótimo da } \\
\text { função }\end{array}$ \\
\hline Downtime & $\mathrm{D}(\mathrm{t})$ & $6 \mathrm{~h}$ & $\begin{array}{c}0,066 \text { do tempo de } \\
\text { inspeção }\end{array}$ \\
\hline Custo & Cost $(\mathrm{t})$ & $16 \mathrm{~h}$ & $\mathrm{R} \$ 3.256$ \\
\hline Disponibilidade & $\mathrm{A}(\mathrm{t})$ & $55 \mathrm{~h}$ & $95,90 \%$ \\
\hline
\end{tabular}

A partir da tabela pode-se observar que não existe uma faixa que contenha os valores de tempo ótimo para todos os parâmetros $\left(t_{D}^{*}, t_{\text {Cost }}^{*}\right.$ e $\left.t_{A}^{*}\right)$ ao mesmo tempo. Assim, cabe agora a tomada de decisão que norteará a política de inspeções e possíveis intervenções da manutenção. Ao optar pelo intervalo de inspeções ótimo de um parâmetro em detrimento a outro, é possível que se tenha uma considerável queda nos valores dos demais parâmetros e assim, a política não chegaria a uma efetividade adequada. Esta disparidade pode ser percebida nas tabelas 2 e 3, que apresentam respectivamente, os valores de cada um dos parâmetros quando utilizando os três diferentes tempos ótimos e suas consequentes variações frente aos valores ótimos de cada um dos parâmetros.

Tabela 2 - Aplicação dos tempos ótimos em cada um dos parâmetros analisados

\begin{tabular}{|c|c|c|c|c|}
\hline $\begin{array}{l}\text { Tempo } \\
\text { ótimo }\end{array}$ & $\begin{array}{c}\text { Valor } \\
\text { (em horas) }\end{array}$ & Downtime & Custo & Disponibilidad \\
\hline$t_{D}^{*}$ & 6 & 0,066 & $\mathrm{R} \$ 4.962,00$ & $83,20 \%$ \\
\hline $\mathrm{t}^{*}{ }_{\text {Cost }}$ & 16 & 0,101 & $\mathrm{R} \$ 3.256,00$ & $93,00 \%$ \\
\hline $\mathrm{t}_{\mathrm{A}}^{*}$ & 55 & 0,262 & $\mathrm{R} \$ 5.322,00$ & $95,90 \%$ \\
\hline \multicolumn{5}{|c|}{ Tabela 3 - Variações dos parâmetros frente aos valores ótimos } \\
\hline $\begin{array}{l}\text { Tempo } \\
\text { ótimo }\end{array}$ & \multicolumn{2}{|c|}{$\begin{array}{c}\text { Variação frente } \\
\text { ao valor ótimo de } \\
\text { downtime }\end{array}$} & $\begin{array}{l}\text { lação frente } \\
\text { lor ótimo de } \\
\text { custos }\end{array}$ & $\begin{array}{c}\text { Variação frente } \\
\text { ao valor ótimo de } \\
\text { disponibilidade }\end{array}$ \\
\hline $\mathrm{t}_{\mathrm{D}}^{*}$ & \multicolumn{2}{|c|}{--} & $34 \%$ & $13 \%$ \\
\hline $\mathrm{t}^{*}{ }_{\text {Cost }}$ & \multicolumn{2}{|c|}{$35 \%$} & -- & $3,0 \%$ \\
\hline $\mathrm{t}_{\mathrm{A}}^{*}$ & \multicolumn{2}{|c|}{$75 \%$} & $39 \%$ & -- \\
\hline
\end{tabular}

Percebe-se a partir da tabela 3 que o intervalo associado ao modelo de custos apresenta variações menores quando comparado aos demais valores ótimos. Esta, por si só, seria uma forte motivação para escolhê-lo como intervalo ótimo para a política de manutenção. Contudo, soma-se ainda o aspecto gerencial que pode ser associado a este intervalo. Visto que a organização analisada apresenta um regime de produção contínuo, com funcionamento 24 horas por dias, sendo dividido em 3 turnos de 8 horas; inspeções a cada 16 horas implicariam em intervenções programadas ao final do segundo turno e antes do início do terceiro turno.

Neste sentido, poder-se-ia aproveitar a presença de duas turmas de manutenção na ação de inspeção (o que poderia inclusive, diminuir o tempo de inspeção $d_{i}$ ), bem como valorizar a troca de experiências e informações entre técnicos destas duas equipes na resolução de problemas ocorridos em turnos anteriores. Ressalta-se aqui, o papel da "memória organizacional" que pode vir a se formar em função desta troca de experiências e eventual registro das intervenções realizadas através de ordens de manutenção, aplicação de check lists e registros de produção (PALMER, 2002). 


\section{CONCLUSÕES}

Após o levantamento e tratamento das informações do processo e posterior implementação da modelagem, verifica-se a aplicabilidade dos conceitos delay time na construção de uma política de inspeções para um sistema de produção contínuo. Como resultado, pode-se estabelecer um intervalo ótimo de 16 horas para as inspeções, implicando assim em uma disponibilidade de $93 \%$ e o menor custo possível frente às opções encontradas para a política preventiva de inspeções. Enxerga-se que os resultados aqui apresentados podem ser utilizados de forma a reestruturar as ações periódicas do departamento de manutenção estudado e; nesse sentido, trazer melhores números em termos reais do processo.

Dada a natureza contínua do processo, fica cada vez mais evidente a importância da qualidade da inspeção e da observação das falhas latentes do processo. Assim, apresenta-se como proposta de trabalhos futuros a adoção de uma modelagem que preveja não só os aspectos aqui abordados, mas também eventuais falhas no processo de inspeção e reparo. Para tanto, sugere-se a extensão da modelagem preconizada por Jones, Jenkinson e Wang (2009) onde se inclui o índice $r$ de qualidade das ações de manutenção. Abordagens similares podem ser encontradas em Berrade et. al (2012) e Zequeira e Bérenguer (2006).

Sugere-se também uma análise de sensibilidade dos parâmetros de custo e duração das intervenções, bem como da taxa de falhas $\lambda$ para um melhor entendimento de como o processo tende a se comportar em função de melhorias de caráter qualitativo (melhoria na qualidade da inspeção) e/ ou quantitativo (redução da taxa de falhas).

Por fim, sugere-se ainda a adoção de uma abordagem multivariada para a tomada de decisão frente ao dilema encontrado na seleção do valor ótimo para o intervalo de inspeções. Visto que cada um dos critérios analisados (downtime, custos e disponibilidade) levou a valores muito díspares quando comparado aos outros critérios, emerge a necessidade de se entender qual dos critérios apresenta maior impacto na gestão da manutenção daquele sistema e assim; caberia ao decisor identificar qual a hierarquia destes parâmetros e sua flexibilidade em termos gerenciais.

Entende-se que este trabalho figura como um primeiro passo na construção de uma prática de gestão de manutenção suportada não só por um melhor levantamento de informações (obtido através da utilização de ERPs e sistemas de gestão de manutenção legados), mas também pela aplicação de modelos matemáticos que reflitam a realidade do contexto estudado. Contudo, este trabalho apresentou algumas limitações na sua realização; pode-se destacar inicialmente o período de análise.

Seja por questões de tempo e acesso ao ambiente de produção, seja pelas informações disponibilizadas pela empresa, a massa de dados analisada refletia apenas a operação da empresa por 4 meses. Neste período, não foi possível observar fatores como sazonalidade ou variações bruscas de demanda (positivas ou negativas) que pudessem impactar na rotina de trabalho da empresa e, por conseguinte, na dinâmica do departamento de manutenção.

Uma outra limitação observada foi o fato de que as informações disponibilizadas pela empresa estavam condensadas, de forma que análises pormenorizadas de cada um dos centros de trabalho da área industrial (produção, subestação, utilidades, etc) não puderam ser realizadas. Percebe-se, entretanto, que esta "aglutinação" não impacta negativamente na análise aqui realizada. Como grande parte das falhas e defeitos ocorre na área de produção e este é o foco inicial da aplicação da política de inspeções, tanto pesquisador quanto empresa entendem que pequenas variações na taxa de falha, nas durações e custos de manutenção não descaracterizariam o contexto investigado. 


\section{REFERÊNCIAS BIBLIOGRÁFICAS}

[1] ALSYOUF, I. The role of maintenance in improving companies' productivity and profitability. International Journal of Production Economics, 105, 70-78, 2007.

[2] BAKER, R. D.; CHRISTER, A. H. Review of delay time OR modeling of engineering aspects of maintenance. European Journal of Operational Research, 73, pp. 407-422, 1994.

[3] BERRADE, M.D.; CAVALCANTE; CRISTIANO A.V.; SCARF, PHILIP A. Maintenance scheduling of a protection system subject to imperfect inspection and replacement. European Journal of Operational Research, 218, 716-725, 2012.

[4] CHRISTER, A. H. Development in delay time analysis for modeling plant maintenance. Journal of the Operational Research Society, 50, pp. 1120-1137, 1999.

[5] CHRISTER, A. H.; WALLER, W. M. Reducing production downtime using delay-time analysis. Journal of Operational Research Society, 35, 499-512, 1984.

[6] CHRISTER, A. H.; WANG, W.; CHOI, K. The delay-time modeling of preventive maintenance of plant given limited PM data and selective repair at PM. IMA Journal of Mathematics Applied in Medicine and Biology, 15, 355-379, 1998.

[7] CHRISTER, A. H.; WANG, W.; BAKER, R. D.; SHARP, J. M. Modeling maintenance practice of production plant using the delay time concept. IMA Journal of Mathematics Applied in Business and Industry, 6, pp. 67-84, 1995.

[8] DEKKER, R. Applications of maintenance optimization models: a review and analysis. Reliability Engineering and System Safety, 51, 229-240, 1996.

[9] ETI, M. C.; OGAJI, S.O.T.; PROBERT, S. D. Strategic maintenance-management in Nigerian industries. Applied energy, 83, 211-227, 2006.

[10] FERREIRA, Rodrigo J. P.; ALMEIDA, Adiel Teixeira de; CAVALCANTE, Cristiano A.V. A multi-criteria decision model to determine inspection intervals of condition monitoring based on delay time analysis. Reliability Engineering and System Safety, 94, 905-912, 2009.

[11] FIGUEIREDO, F. A.; CAVAlCANTE, C. A. V.; ALMEIDA, A. T. Preventive maintenance planning using prior expert knowledge and multicriteria method PROMETHEE III. Proceedings of European Safety and Reliability Conference (ESREL)2008, Valência, 2008.

[12] JONES, B.; JENKINSON, I.; WANG, J. Methodology of using delay-time analysis for a manufacturing industryThe use of Bayesian network modelling for maintenance planning in a manufacturing industry. Reliability Engineering and System Safety, 94, 111-124, 2009.

[13] LEVITT, J. The Handbook of Maintenance Management. Nova Iorque; Industrial Press INC., 1997.

[14] The Complete Guide to Preventive and Predictive Maintenance. Nova Iorque; Industrial Press INC. 2003.

[15] MOWBRAY, J. Reliability Centred Maintenance. Londres; Butterworth-Heineman. 1997.

[16] MURTHY, D. N. P.; ATRENS, A.; ECCLESTON, J. A. Strategic maintenance management. Journal of Quality in Maintenance Engineering, vol. 8, nº 4, 287-305, 2002. 
[17] NASCIMENTO NETO, L. P.; ARAÚJO, H. A. B. T.; LOPES, R. S.; GOMES, G. R. S.G.; CAVALCANTE, C. A. V. Uso de análise snapshot e do conceito delay time para definição de tempos de inspeção: Um estudo de caso para o planejamento de manutenção de frotas. Anais do XXXII Encontro Nacional de Engenharia de Produção, Bento Gonçalves - RS, 15 a 18 de Outubro de 2012.

[18] PALMER, R. Maintenance work order planning. In: Maintenance Engineering Handbook. HIGGINS, L. R.; MOBLEY, R. K. (editors). 6. ed. McGraw-Hill: 2002.

[19] SCARF, P. A. On the application of mathematical models in maintenance. European Journal of Operations Research, 99, 493-506, 1997.

[20] TSANG, Albert H.C. Strategic dimensions of maintenance management. Journal of Quality in Maintenance Engineering, vol. 8, nº 1, 7-39, 2002.

[21] WANG, H. Subjective estimation of the delay time distribution in maintenance modeling. European Journal of Operations Research, 99, 516-529, 1997. A survey of maintenance policies of deteriorating systems. European Journal of Operations Research, 43, 471-476, 2002. A delay time based approach for risk analysis of maintenance activities. The Journal of the Safety and Reliability Society, 23, 103-113, 2003.

[24] WANG, W.; JIA, X. An Empirical Bayesian Based Approach to Delay Time Inspection Model Parameters Estimation Using Both Subjective and Objective Data. Quality and Reliability Engineering International, 23, pp. 95-105, 2007.

[25] ZEQUEIRA, R. I.; BÉRENGUER, C. Periodic imperfect preventive maintenance with two competing failure modes. Reliability Engineering and System Safety, 91, 460-468, 2006. 